DOI: https://doi.org/10.46296/yc.v5i8edespmar.0135

\title{
DIAGNÓSTICO PARA DESARROLLAR UN SISTEMA DE GESTIÓN DE CALIDAD PARA LA PROMOCIÓN CULTURAL DE JULCUY
}

\section{DIAGNOSIS TO DEVELOP A QUALITY MANAGEMENT SYSTEM FOR THE CULTURAL PROMOTION OF JULCUY}

\author{
Chilán-Choez Omar Onofre ${ }^{1}$; Verduga-Pino Alexandra ${ }^{2}$; Vivas-Vivas Esperanza ${ }^{3}$ \\ 1 Universidad Técnica De Manabí. Portoviejo, Ecuador. Correo: ochilan3164@utm.edu.ec. \\ ${ }^{2}$ Docente Investigador de la Facultad de Ciencias Administrativas y Económicas de la \\ Universidad Técnica De Manabí. Portoviejo, Ecuador. Correo: alexandra.verduga@utm.edu.ec. \\ ORCID ID: https://orcid.org/0000-0003-4864-0499. \\ ${ }^{3}$ Coordinadora del Programa de Maestría con Trayectoria de Investigación en Gerencia de la \\ Calidad e Innovación de la Universidad Técnica de Manabí. Portoviejo, Ecuador. Correo: \\ fe.vivas@utm.edu.ec. ORCID ID: https://orcid.org/0000-0003-2382-1808.
}

\begin{abstract}
Resumen
La última década Ecuador ha incrementado las intenciones de cada sector económico y productivo, respecto al establecimiento de políticas de desarrollo para fomentar el crecimiento sostenible; al impulsar el sector terciario de la industria cultural se influye positivamente en subsectores como el turismo, especialmente en el área rural que se enfoca por establecer proyectos de promoción cultural que atraigan capitales externos a las comunidades, sean de origen nacional o extranjeros. La parroquia Julcuy del cantón Jipijapa, es parte del mapa patrimonial de la provincia de Manabí, sin embargo presenta como problemática la falta de promoción cultural que enfoque sus atributos naturales, flora, fauna y muchas otras riquezas como la arqueología precolombina con sus vestigios de las civilizaciones Valdivia, Machalilla y Manteña, que se encuentran en la comunidad y pozos de agua azufrada y cuevas ancestrales, entre otros; este estudio se centra en el desarrollo de un diagnóstico para la elaboración de un sistema de gestión de la calidad (SGC) para la promoción cultural de la parroquia Julcuy del cantón Jipijapa de la provincia de Manabí, basado en los diferentes atractivos turísticos que presenta esta zona, utilizando métodos como son el deductivo, inductivo, estadísticos, analítico y técnicas como la observación, encuesta y entrevista que concluye con un diagnóstico para desarrollar un SGC para la promoción cultural que permitiría mostrar de manera diversificada su herencia cultural a los visitantes y con ello la generación de nuevas fuentes de empleo en comunidades rurales.
\end{abstract}

Palabras claves: Industria cultural, sector terciario, promoción cultural, sistema de gestión de la calidad, diagnóstico.

\begin{abstract}
The last decade Ecuador has increased the intentions of each economic and productive sector, regarding the establishment of development policies to promote sustainable growth; By promoting the tertiary sector of the cultural industry, subsectors such as tourism are positively influenced, especially in rural areas, which is focused on establishing cultural promotion projects that attract external capital to the communities, whether of national or foreign origin. The Julcuy parish of the Jipijapa canton is part of the heritage map of the Manabí province, however, it presents as a problem the lack of cultural promotion that focuses on its natural attributes, flora, fauna and many other riches such as pre-Columbian archeology with its vestiges of the Valdivia, Machalilla and Manteña civilizations, found in the community and sulfur water wells and ancestral caves, among others; This study focuses on the development of a diagnosis for the elaboration of a quality management system (QMS) for the cultural promotion of the Julcuy parish of the Jipijapa canton of the Manabí province, based on the different tourist attractions that this area, using methods such as deductive, inductive, statistical, analytical and techniques Información del manuscrito:
\end{abstract}

Fecha de recepción: 18 de enero de 2021.

Fecha de aceptación: 30 de marzo de 2021.

Fecha de publicación: 31 de marzo de 2021 
such as observation, survey and interview that concludes with a diagnosis to develop a QMS for cultural promotion that would allow visitors to show their cultural heritage in a diversified way and with This will generate new sources of employment in rural communities.

Keywords: Cultural industry, third sector, cultural promotion, quality management system, diagnosis.

\section{Introducción}

En la actualidad, las sociedades se encuentran

en constante transformación y en igual sentido lo hace industria cultural (Unesco, 2021); el concepto Industria Cultural fue desarrollado por Theodor Adorno y Max Horkheimer, orientado a los bienes y servicios culturales cuyo valor económico fundamental deriva de su valor cultural y tienen el potencial para la creación de riqueza y empleo a través de la generación y aprovechamiento de la propiedad intelectual y aunque en un principio fue utilizado en singular, actualmente el termino es en plural: Industrias Culturales, que se insertan en el sector terciario de la economía como lo es el turismo, el campo cultural y el desarrollo del sector de la industria cultural, está constituido por un moderno mercado cultural de masas siendo uno de los factores más decisivos que permiten que se desarrolle (Brunner \& Catalán, 1987).
Siendo que la cultura es un conjunto de los rasgos distintivos, espirituales, materiales y afectivos que caracterizan una sociedad 0 grupo social desde la definición de La UNESCO (2015) también las artes y las letras, los modos de vida, los derechos fundamentales del ser humano, los sistemas de valores, creencias y tradiciones también la conforman, (Carmen Ballesteros, 2018) ; Mientras que para Batallas (2014) "la cultura, entendida como un producto humano, es creación humana que se ha dado a lo largo de la historia en una constante relación con la naturaleza y se relaciona con los símbolos, los sentidos y significaciones de lo que un determinado grupo de hombres realiza; es la forma de entender la vida y la muerte" (p. 32)

En el Ecuador, la cultura, constituye el conjunto de características y modos de vida y de desarrollo que definen a un grupo social y que, 
tratada desde la visión de la gestión cultural, la misma cimienta su accionar en la administración de todos los recursos disponibles que le permite diseñar, crear y difundir un bien cultural (BCE, 2004), que en este trabajo refiere al patrimonio cultural y ancestral, se requiere para este fin, contar con políticas públicas que son las normativas que el Estado genera e implementa para alcanzar la solución a las diferentes necesidades que tiene la sociedad.

El turismo como sector terciario de la economía es la actividad que se desarrolla gracias a la evolución de las formas laborales en las sociedades desarrolladas y en vías de desarrollo, siendo su objetivo el descanso, la diversión y el conocimiento de otras formas de vida (Quesada, 2010) además las herencias culturales que hacen conexión con el campo de estudio de este diagnóstico como lo es la promoción cultural y que permite ampliar el nutrido acervo bibliográfico del mismo.

El objeto del presente trabajo es desarrollar un sistema de gestión de calidad para la promoción cultural de Julcuy a través de un análisis que permite diagnosticar nociones teóricas sobre promoción cultural y patrimonio ancestral como elementos esenciales para el trabajo en las comunidades rurales.

Entender la riqueza de las tradiciones, formas de vida $y$ potencialidades para la preservación de un patrimonio cultural; desde el desarrollo de acciones con un enfoque evolutivo de cada uno de los elementos inmateriales que forman parte del mismo constituyen la razón esencial de esta investigación que con el presente diagnostico se convierte en una herramienta para desarrollar un SGC que salvaguarde las tradiciones culturales de Julcuy y apertura opciones para el desarrollo de la localidad alineándose a los componentes constitucionales que le preceden a los procesos de desarrollo de la cultura.

"La calidad como excelencia supone lograr el compromiso de todos los integrantes de la organización para lograr un producto lo mejor posible, empleando los mejores componentes, la mejor gestión y los mejores procesos posibles". (Francisco J. Miranda Gonzalez, Antonio Chamarro Mera \& Sergio Rubio Lacoba, 2007) 
Según (Yánez, Carlo M., 2008) que "Un Sistema de Gestión de la Calidad es una forma de trabajar, mediante la cual una organización asegura la satisfacción de las necesidades de sus clientes. Para lo cual planifica, mantiene y mejora continuamente el desempeño de sus procesos, bajo un esquema de eficiencia y eficacia que le permite lograr ventajas competitivas".

La gestión cultural para su implementación se apoya necesariamente en lineamientos $y$ estrategias públicas y especialmente en la utilización de todos los recursos que disponga entre ellos: humanos, técnicos, tecnológicos y financieros, que permitan el desarrollo del quehacer cultural, la participación en actividades culturales, la ejecución de proyectos, de programas, y más.

La gestión cultural debe responder a la realidad de la localidad, por lo tanto, las directrices que se establezcan tendrán que estar definidas en base a las características e individualidades de la población ecuatoriana. En el caso del Ecuador, los procesos de gestión y desarrollo cultura está delimitada en la Constitución del
2008, constituyéndose así también en el basamento de políticas sectoriales que en igual sentido están diseñadas para orientar las acciones que en el caso del área cultural se ejecutan para establecer cambios sociales, económicos y políticos que contribuyen a fortalecer el desarrollo cultural del país.

"En los últimos años se han incrementado las intenciones de cada sector, al menos en el área rural por desarrollar proyectos turísticos que atraigan capitales externos a la comunidad, sean de origen nacional o mucho mejor en el caso de capitales extranjeros" (Hernández Rueda \& Monsalve Castro, 2015).

En la provincia de Manabí del Cantón Jipijapa encontramos ubicada la Parroquia Julcuy la principal problemática es la falta de innovación a la promoción cultural y el turismo rural y en la actualidad es apreciado por sus atributos naturales, flora y fauna y con muchas otras riquezas que se encuentran en la comunidad.

En la parroquia Julcuy hay un hermoso valle en el cual está 
incluido el Cerro de Julcuy uno de los más grande de la zona y variedad de especies animales, también se encuentra senderos eco turísticos como son el Tigrillo en donde contamos con pozos de agua azufrada y cuevas ancestrales, un hermoso rio, restos arqueológicos encontrados en estos últimos años, y sus diversos platos típicos como el seco de chivo y vía a los recinto Mero Seco, Piñas, Carrizal, Soledad y Agua blanca en donde se practica ciclismo de montaña y caminatas.

\section{Desarrollo}

\subsection{Calidad y desarrollo sustentable}

El abordaje de la problemática de la calidad en relación a la promoción cultural de una localidad, implica no sólo el análisis de la satisfacción de los turistas con los distintos servicios, entendida como Calidad Total, sino también la contribución del turismo al desarrollo sostenible de los destinos turísticos y la promoción de la cultura de la localidad. Estos dos componentes constituirían la calidad global.
Almache Martínez, (2013) da a conocer que en un territorio se produzca desarrollo es importante la confluencia de un conjunto de factores (capitales) que interaccionan en un espacio produciendo una sinergia que conduciría al desarrollo: capital financiero, redes de empresas y de organizaciones civiles basadas en la confianza, identidad con un territorio, sistemas de innovación, cuidado ambiental y conservación del patrimonio, redes de comunicación, etc.

De este modo, el desarrollo cultural de una localidad debe estar enfocado a la satisfacción de tres actores sociales fundamentales que intervienen en el proceso: turistas, empresarios y residentes. Donde el turista buscará fundamentalmente la satisfacción de su experiencia de viaje, el empresario buscará fundamentalmente la rentabilidad del negocio, y el residente buscará, mediante la actividad turística, elevar el nivel y la calidad de vida de la población.

En la concepción y práctica del desarrollo resulta necesario diferenciar entre actor y agente de desarrollo. Los actores locales 
pueden ser individuos, grupos 0 instituciones cuyo sistema de acción coincide con los límites de la sociedad local, pueden estar ligados a la toma de decisiones (políticos institucionales), ligados a técnicas particulares (expertos profesionales), o ligados a la acción sobre el terreno (la población y sus expresiones activas). (Guerrero Coloma, 2015, pág. 87)

Bonilla Velástegui, (2016) expresa que en tanto los agentes de desarrollo son aquellos actores sociales portadores de propuestas políticas, económicas, sociales y culturales que tienden a capitalizar mejor las oportunidades locales. Es decir, tienen "poder" para generar un efecto sobre la actividad turística, con capacidad para intervenir, modificar o influir el curso del sector. Por otra parte, y en función de este modelo de desarrollo sustentable, el objetivo primordial será contribuir al desarrollo del territorio y de la comunidad, a través de una más plena satisfacción del usuario (turista y residente).

Hoy en día se presume que este usuario demanda cada vez más y mejores servicios y que la diversificación de la oferta turística es inducida por el aumento de sus exigencias. De este modo, el usuario debiera convertirse en el centro de atención de las empresas que conforman la estructura económica productiva de un destino turístico.

La dinámica del desarrollo se fundamenta en la reproducción constante del capital sinérgico, concepto que hace referencia a la capacidad de una sociedad local para articular provechosamente sus recursos endógenos.

Las formas de capital que puede detentar un territorio incluyen recursos tanto materiales como intangibles, donde se destaca el capital institucional y capital social y cultural junto al natural, económico, cognitivo, simbólico, cívico y humano. El aporte principal de este enfoque es la relevancia que se otorga a la voluntad organizada de una sociedad para valorizar y aprovechar sus capacidades. (Hernández Rueda \& Monsalve Castro, 2015, pág. 116)

Capacidades que dependen en buena medida de factores socios institucionales y culturales. El concepto de desarrollo no dispone 
de un cuerpo teórico homogéneo, por el contrario, existen diversas perspectivas económicas y sociales.

En el campo del turismo, la Organización Mundial del Turismo (OMT) adoptó el enfoque sostenible para el turismo y aplica los principios de desarrollo sostenible en todos sus estudios de planificación y desarrollo. En este sentido la OMT ha definido el desarrollo sostenible de la siguiente manera.

Pailiacho Mena, (2013) considera que el desarrollo sostenible atiende a las necesidades de los turistas actuales $y$ de las regiones receptoras y al mismo tiempo protege y fomenta las oportunidades para el futuro. Se concibe como una vía hacia la gestión de todos los recursos de forma que puedan satisfacerse las necesidades económicas, sociales y estéticas, respetando al mismo tiempo la integridad cultural, los procesos ecológicos esenciales, la diversidad biológica y los sistemas que sostienen la vida. (pág. 36)

Las directrices para el desarrollo sostenible del turismo y las prácticas de gestión sostenible son aplicables a todas las formas de turismo en todos los tipos de destinos, incluidos el turismo de masas y los diversos segmentos turísticos. Los principios de sostenibilidad se refieren a los aspectos ambiental, económico y sociocultural del desarrollo turístico, habiéndose de establecer un equilibrio adecuado entre esas tres dimensiones para garantizar su sostenibilidad a largo plazo.

Si bien algunos de estos enfoques del desarrollo incorporan la dimensión sociocultural, se advierte una ausencia de la consideración de la persona, como fin último de cualquier proceso de desarrollo, obviando la cuestión de los valores en juego. Amaya León, (2011) considera que "las nociones sucesivas de progreso, crecimiento, expansión, propias de una concepción del desarrollo económico, y prioritariamente consumista, excluyen una concepción más comprehensiva del desarrollo humano, necesarias para una concepción integral".

La noción de desarrollo a la que se apela, debiera rescatar a la persona y a los valores éticos. El desarrollo es el acceso a un modo de vida en 
la que la persona tiene más importancia que la producción de bienes y servicios y en el que la realización personal y colectiva se prioriza sobre su función de consumidor.

De este modo, el desarrollo no sólo depende de los grandes sistemas macroeconómicos ni de las instituciones centralizadas, sino también de los micros iniciativas y la microeconomía que radica en producir y consumir que responden a otros objetivos, a otros valores, haciendo que las actividades de producción contribuyan a mejorar la calidad de las relaciones sociales.

Desde una perspectiva sociocultural del desarrollo turístico se encuentra condicionado a los procesos de construcción simbólica y material de los diferentes actores. "Así, las personas y su subjetividad son un requisito indispensable del desarrollo y reconocerla significa potenciar la capacidad para manejar los procesos de desarrollo turístico local" (Barona López, Estévez Bonilla, Tabares Burbano, \& Paredes Cruz, 2018, pág. 213).

Asimismo, el tipo de capital social de una comunidad definirá comportamientos facilitadores 0 limitadores del desarrollo turístico, es por ello que el análisis del estado de ese capital social es un aspecto fundamental a tener en cuenta al evaluar el aporte del turismo al desarrollo sustentable.

\subsection{Los Sistemas de Gestión de} Calidad

La implementación de Sistemas de Gestión de Calidad consiste en la aplicación de herramientas de gestión modernas, reconocidas internacionalmente, y enfocadas en la satisfacción del cliente y la mejora continua de los procesos que los organismos, las organizaciones y las empresas llevan a cabo en el marco de sus procesos de producción de bienes y servicios. El objetivo final al aplicar criterios de calidad es elevar la competitividad en pos del desarrollo sustentable de los distintos sectores.

"Los Sistemas de Gestión de Calidad son documentos de aplicación voluntaria que contienen especificaciones técnicas extraídas de la experiencia y los avances de la tecnología, y deben satisfacer determinados requerimientos, entre otros los descriptos en las normas" 
(Guadalupa Guerrero, 2012, pág. 54).

Una norma es un documento, establecido por consenso y aprobado por un organismo reconocido en actividades de Normalización como lo es IRAM (Instituto Argentino de Normalización y Certificación) en nuestro país. La norma establece, para usos comunes y repetitivos, reglas, criterios o características para las actividades 0 sus resultados, procurando la obtención de un nivel óptimo de ordenamiento en un contexto determinado.

Entre las normas de mayor aplicación en todo el mundo (actualmente con más de 1.000.000 certificados en todo el mundo), se encuentran las ISO serie 9000 , que describe los fundamentos de los sistemas de gestión de la calidad y especifica la terminología para los sistemas de gestión de la calidad.

Mendoza Torres, (2017) expresa que son normas que surgieron para el logro del aseguramiento de calidad que, en su origen, estaban orientadas a empresas de manufactura, tras ser revisadas en 1994 y 2000, fueron actualizadas para cubrir todos los aspectos de la gestión de la calidad de organizaciones de todo tipo y tamaño, incluyendo las prestadoras de servicio.

La ISO 9001 especifica los requisitos para los sistemas de gestión de la calidad aplicables a toda empresa que necesita demostrar su capacidad para proporcionar productos que cumplan los requisitos de sus clientes y los reglamentos que le sean de aplicación. Otro ejemplo es las ISO 14000 para Sistemas de Gestión Ambiental. Y para demostrar la creciente importancia y expectativa en relación a los beneficios de normalización para las actividades turísticas, esta organización internacional ISO, asume, en enero de 2005, el desafío de crear el ISO/TC 228 Tourism and related services.

\subsection{La gestión cultural.}

"La gestión refleja un corpus de teorías, de conocimientos y de métodos que tiene de la economía, de las humanidades, de las ciencias sociales, del marketing, de las ciencias de la administración, de las 
finanzas, etc." (Merino Jaramillo, 2014, pág. 83).

Se relaciona con la administración de todos los recursos disponibles, sean económicos, técnicos 0 humanos, lo que le permitan planificar, organizar, ejecutar, evaluar y hacer seguimiento de procesos culturales; así como ser utilizada en todas las áreas relacionadas con la actividad cultural de las mismas, que van desde lo técnico-administrativo hasta lo legal y económico.

Su fin es la consolidación de objetivos a corto, mediano y largo plazo de acuerdo a la planificación realizada para los mismos. Se requiere de canales de comunicación para su respectiva socialización.

La gestión cultura a través de su accionar genera propuestas y contribuye a solucionar los requerimientos relacionados con el sector cultural. Entre sus lineamientos está el incentivar la participación, la creatividad, el consumo y acceso a los productos culturales; siendo necesario para ello la optimización en la ejecución, monitoreo y evaluación de todos los procesos y de las actividades planificadas, ya que, con ello, garantiza los derechos culturales de la ciudadanía, así como coadyuva al fortalecimiento y promoción del quehacer cultural del país.

Entre los objetivos que tiene la Gestión Cultural está el incentivar la creación, circulación, promoción y consumo de las diferentes manifestaciones culturales, requiriendo para su ejecución de la participación y apoyo profesional de gestores culturales, quienes a través de sus competencias, también contribuyen a fortalecer, posicionar y promocionar los productos $\mathrm{O}$ servicios culturales tanto a nivel nacional como internacional; por lo tanto, su gestión es indispensable para desarrollar estrategias de relación entre el actor, el gestor cultural, la institucionalidad cultural y la sociedad.

Sin embargo, tanto la gestión cultural como el rol que desempeñan los gestores culturales, no han sido debidamente tratados por la institucionalidad cultural del país; haciéndose indispensable observar el rol y las funciones que tiene el gestor 
cultural ya que de la ejecución de las mismas depende la inclusión y la promoción del sector cultural del país a nivel nacional e internacional.

Pazmiño Ortiz, (2018) considera que la falta de importancia que se le ha dado al rol que tiene el gestor cultural ha generado la desarticulación de este en lo que se refiere a su accionar con respecto a la institucionalidad, incidiendo indiscutiblemente, en el desarrollo de sus funciones, las mismas que encuentran delineadas en las normativas públicas culturales. (pág. 95)

Los lineamientos que ejecutan los Estados en lo que se refiere al quehacer cultural, a su desarrollo e implementación, garantizando los derechos culturales de la sociedad, pueden considerados como gestión pública cultural.

Otra manera de hacer gestiones culturales en la que intervienen tanto la institucionalidad cultural como la gestión privada, la misma que se desarrolla conjuntamente con el apoyo gubernamental, aunque el proceso de ejecución de los proyectos 0 actividades culturales las realiza la gestión privada.

La gestión cultural en el Ecuador actualmente tiene serios cuestionamientos tanto por parte de la sociedad, así como de los gestores culturales en particular ya que consideran que aún no se observan cambios notorios en el sector cultural. Esto inclusive después de la promulgación de la Ley Orgánica de Cultura y de la implementación de las directrices generadas para el área cultural pues manifiestan que no existe una adecuada comunicación con las instancias culturales y la ciudadanía, que hace falta la socialización de las funciones, proyectos y actividades que tienen planificadas las nuevas dependencias culturales, determinando con ello que no existe mayor participación de actores y gestores culturales en las mismas, lo que hace evidente que no se llega a apoyar a la ciudadanía, de manera equitativa e igualitaria.

\subsection{Modelo de gestión turística}

Antes de empezar a hablar de que es un modelo de gestión primero es necesario hacer mención al turismo 
como "un sistema complejo de servicios, que necesita de soportes físicos, servicios y turistas; por su gran complejidad necesita constantemente del diseño de modelos de gestión que permitan mejorar el desarrollo de la actividad turística estructurando estrategias e indicadores" (Romero Donoso, 2016).

El modelo de gestión turística es una herramienta guía que permite mejorar el desarrollo de una actividad en este caso la actividad turística. La estructuración de un modelo de gestión nace del agotamiento de estrategias, por presencia de crisis, por el desequilibrio en el sistema de gestión, por el desequilibrio tecnológico, medio ambiental y espacial lo que deja claro que la gestión turística es la base para el desarrollo sostenible de cualquier destino turístico utilizando cualquiera de sus modalidades.

Un "modelo de gestión no es más que el diseño de estrategias que se sustentan en un sistema de indicadores que permiten orientar la toma de decisiones para conseguir los objetivos fijados" (Sarminto Iglsias, 2012) por lo que es posible dar a conocer que el mismo puede estar estructurado de la siguiente manera: además estos autores muestran cómo se estructura un modelo de gestión.

El modelo de gestión turística busca "mejorar y modernizar la oferta ante los nuevos escenarios, direccionándose hacia un nuevo modelo de desarrollo turístico" (Bonilla Velástegui, 2016), es necesario analizar los cambios de la demanda, cuáles son los nuevos gustos, preferencias y hacer la clasificación de las nuevas tipologías de los clientes potenciales y futuros.

Desde "el surgimiento del turismo se han presentado diversas metodologías y herramientas que permitan analizar y gestionar al turismo de masas que surgió en la segunda mitad del siglo $X X$, presentándose como una actividad de gran dinamismo, con enfoques en los social y económico" (Pailiacho Mena, 2013).

En la actualidad el turismo ha tomado un giro debido a los problemas que lleva consigo la implementación del mismo, existen nuevos modelos de gestión que 
permiten mejorar las acciones del y que constituya una ventaja competitiva para las empresas y los destinos turísticos, y no es más que una guía con diferentes indicadores de gestión, adquiriendo la importancia a través de políticas públicas turísticas.

"Las iniciativas turísticas están necesitadas de modelos de desarrollo turísticos, adecuados a las características del medio" (Mendoza Torres, 2017). Refiriéndose al turismo rural surgen los factores socioeconómicos, culturales ambientales, componentes del desarrollo sustentable, los mismos que deben ser tomados en cuenta en función a la realidad de su entorno.

Por lo tanto, los nuevos modelos de gestión y desarrollo turístico sustentable deben contribuir al principal objetivo que esta sintetizado en un solo objetivo como lo es el mejoramiento de la calidad de vida de las personas de un determinado territorio.

"La elaboración de modelos de gestión en el desarrollo turístico local permite la generación de ideas acerca de nuevos productos 0 servicios de acorde al entorno en el que se encuentra" (Almache Martínez, 2013); lo que aporta a la visualización de estrategias con un enfoque progresivo. La defensa de los modelos turísticos de baja intensidad (en cuanto a los impactos ocasionados) coincide con el creciente interés en los enfoques del desarrollo rural; lo que favorece al control y dirección local además de la aplicación de estrategias basados a la sostenibilidad de acuerdo al desarrollo económico, social y ambiental.

Para diseñar el modelo de gestión es necesario determinar los procesos, analizar las personas y determinar que tecnología usar, presentándose estos tres como los pilares del modelo de gestión; también se deben analizar los mecanismos externos privados, los mecanismos internos (operación) y los mecanismos externos regulatorios.

Para entender los principios de gestión de los destinos turísticos, hay que tener clara previamente la naturaleza de la actividad turística a la cual deben adaptarse. La naturaleza de la actividad turística como toda actividad económica, es 
posible analizarla desde dos puntos de vista: el de la oferta y el de la demanda.

En la medida en que su confluencia en el mercado se produzca armónicamente, se alcanzará la eficiencia. "Además, para poder lograr una visión integradora del proceso de gestión del destino turístico como unidad analítica esencial del turismo e introducirse en el análisis del modelo, es imprescindible analizar el fenómeno turístico de manera más general, a partir del modelo que describen su estructura funcional" (Merino Jaramillo, 2014).

\section{Metodología}

Considerando las características que guarda el presente estudio se consideró la modalidad no experimental con la finalidad de estudiar las variables sin modificarlas, de la misma manera se consideró que el tipo de investigación seria bibliográfica, debido a que se recolecto información teórica del tema tratado como es el sistema de gestión de la calidad para la promoción cultural. Del mismo modo, también fue descriptiva porque se centró en los datos mediante encuestas a la población objetiva de este estudio.

Como población se seleccionó a las autoridades parroquiales y personal que labora en el Gobierno Autónomo Descentralizado de la Parroquia Julcuy del Cantón Jipijapa de la Provincia Manabí (20 miembros) al que se le aplicaron los instrumentos de recolección de información.

Los métodos utilizados fueron el Inductivo - Deductivo con el fin de estudiar problemática de la investigación tanto a nivel nacional, así como a nivel cantonal, además que permitió la generación de conclusiones especificas por medio del proceso de recolección de información de fuentes primarias, así como secundarias.

Como segundo método se consideró el Análisis - Síntesis mismo que permitió el análisis e interpretación de los resultados obtenidos por medio de los instrumentos de encuesta, el cual fue aplicado a la población seleccionada. 


\section{Resultados}

Con la finalidad de saber cómo el Gobierno

Autónomo

Descentralizado de la Parroquia Julcuy desarrolla las actividades que se relacionan con la promoción cultural se aplicó el instrumento de encuesta con la finalidad de desarrollar el correspondiente diagnostico para establecer la necesidad de desarrollar un Sistema de Gestión de Calidad para la promoción cultural de Julcuy, proceso por medio del cual se obtuvieron los siguientes resultados:

Tabla 1: Resultados del instrumento de encuesta aplicado al personal que labora en el Gobierno Autónomo Descentralizado de la Parroquia Julcuy.

\begin{tabular}{|c|c|c|c|c|c|c|}
\hline \multirow{2}{*}{ Preguntas } & \multicolumn{2}{|c|}{ De acuerdo } & \multicolumn{2}{|c|}{ En desacuerdo } & \multicolumn{2}{|c|}{ Indeciso } \\
\hline & $F$ & $\%$ & $F$ & $\%$ & $\mathbf{F}$ & $\%$ \\
\hline $\begin{array}{l}\text { ¿Considera que el patrimonio } \\
\text { cultural (creencias, tradiciones, } \\
\text { costumbres) de la parroquia Julcuy } \\
\text { debe ser fomentado y conservado para } \\
\text { potenciar el turismo }\end{array}$ & 20 & 100 & 0 & 0 & 0 & 0 \\
\hline $\begin{array}{l}2 \text { ¿Considera que los sitios y lugares } \\
\text { turísticos de la parroquia Julcuy han } \\
\text { sido preservados? }\end{array}$ & 3 & 15 & 17 & 85 & 0 & 0 \\
\hline $\begin{array}{l}3 \text { ¿Considera que algunos lugares de la } \\
\text { parroquia Julcuy son de interés } \\
\text { turístico? }\end{array}$ & 20 & 100 & 0 & 0 & 0 & 0 \\
\hline $\begin{array}{l}\text { 4. ¿Considera que la atención de los } \\
\text { prestadores de servicios es la } \\
\text { apropiada para realizar turismo? }\end{array}$ & 4 & 20 & 13 & 65 & 3 & 15 \\
\hline $\begin{array}{l}\text { 5. ¿Considera que los productos de la } \\
\text { comunidad están a la altura de otros } \\
\text { ofertados en el cantón? }\end{array}$ & 7 & 35 & 3 & 15 & 10 & 50 \\
\hline $\begin{array}{l}\text { 6. ¿Considera que con la apropiada } \\
\text { gestión, la parroquia Julcuy puede } \\
\text { potencializar el turismo cultural? }\end{array}$ & 20 & 100 & 0 & 0 & 0 & 0 \\
\hline $\begin{array}{l}\text { 7. ¿Considera Ud. que las entidades } \\
\text { públicas están involucradas en el } \\
\text { desarrollo turístico de la parroquia } \\
\text { Julcuy? }\end{array}$ & 6 & 30 & 12 & 60 & 2 & 10 \\
\hline $\begin{array}{l}\text { 8. ¿Considera que la comunidad es un } \\
\text { lugar de interés para hacer turismo? }\end{array}$ & 20 & 100 & 0 & 0 & 0 & 0 \\
\hline $\begin{array}{l}\text { 9. ¿Considera que los servicios } \\
\text { ofrecidos (alojamiento, alimentación) } \\
\text { son apropiados? }\end{array}$ & 3 & 15 & 11 & 55 & 6 & 30 \\
\hline $\begin{array}{l}\text { 10. ¿Considera que las personas de la } \\
\text { parroquia Julcuy están capacitadas } \\
\text { para realizar actividades turísticas? }\end{array}$ & 2 & 10 & 13 & 65 & 5 & 25 \\
\hline
\end{tabular}

Fuente: Instrumento de encuesta aplicado al personal que labora en el Gobierno Autónomo Descentralizado de la Parroquia Julcuy

Elaborado por: Autor. 
Grafico 1: Resultados del instrumento de encuesta aplicado al personal que labora en el Gobierno Autónomo Descentralizado de la Parroquia Julcuy.

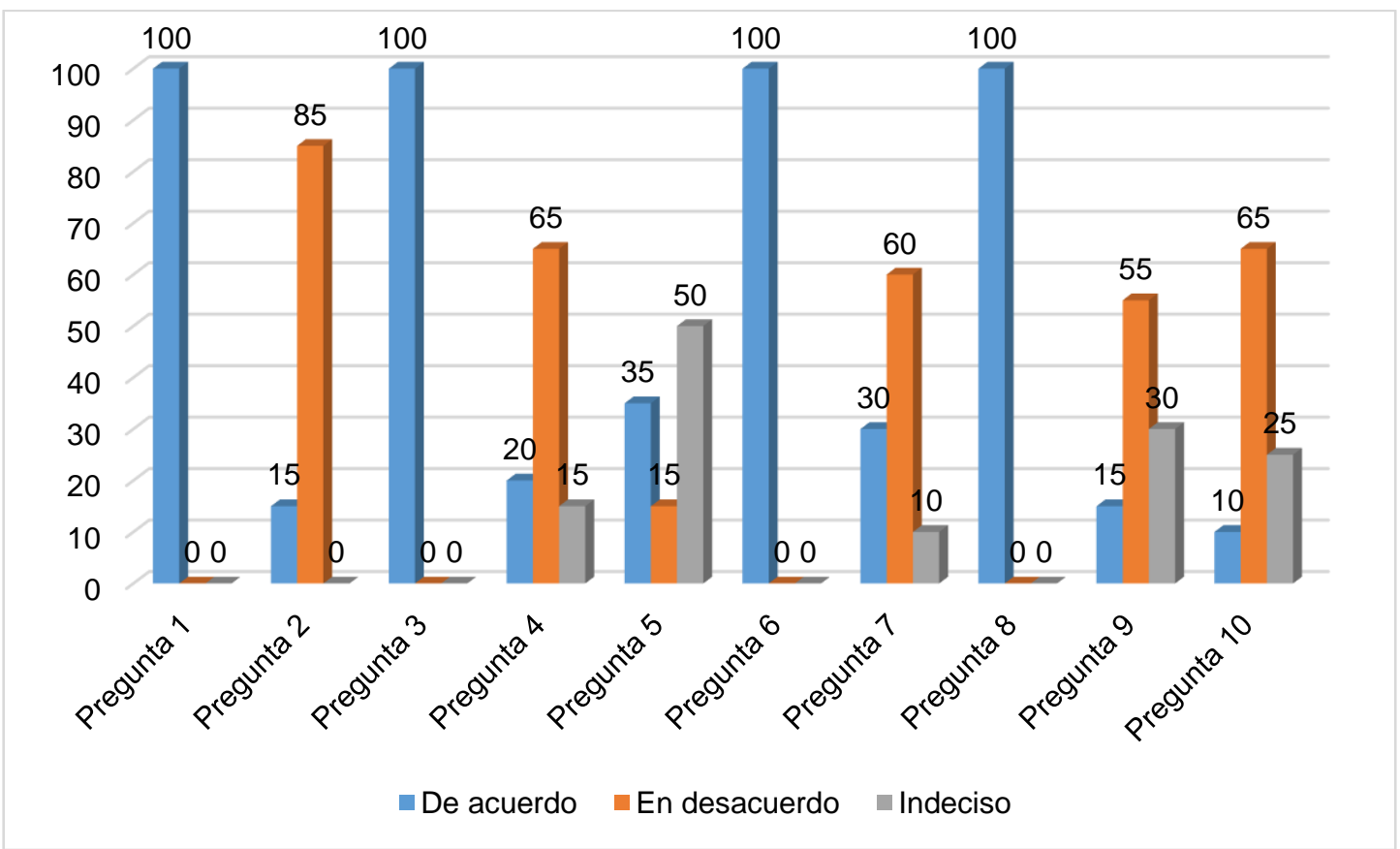

Elaborado por: Autor.

\section{Análisis e interpretación:}

Del proceso de recolección de información en la pregunta uno fue posible establecer que el $100 \%$ del personal encuestado considera que el patrimonio cultural de la parroquia Julcuy debe ser fomentado y conservado para potenciar el turismo.

En lo que refiere a la pregunta dos fue posible determinar que el $15 \%$ de los encuestados consideran que los sitios y lugares turísticos de la parroquia Julcuy se han preservado de forma oportuna, y el $85 \%$ restante expreso estar en desacuerdo con esto y consideran que no se han aplicado acciones apropiadas para la preservación de los lugares turísticos de la localidad.

Por medio de la pregunta tres fue posible determinar que el $100 \%$ del personal encuestado consideran que los lugares de la parroquia Julcuy son de interés turístico, a pesar de ello del GAD parroquial de Julcuy no ha desarrollado estrategias para la preservación de los mismos.

Mediante la pregunta cuatro se estableció que el $20 \%$ de los encuestados consideran que la atención de los prestadores de servicios si es la apropiada para 
realizar turismo, el 65\% expreso estar en desacuerdo, debido a que esta presenta falencias en cuanto a servicios se refiere; el $15 \%$ restante expreso no conocer de forma oportuna la realidad de estos servicios.

Respecto a la pregunta cinco es posible mencionar que el $35 \%$ de los encuestados expresaron estar de acuerdo con que los productos de la comunidad están a la altura de otros ofertados en el cantón; el 15\% expreso estar en desacuerdo y el $50 \%$ respondió estar indeciso en lo que refiere a los productos culturales y turísticos de la localidad.

Por medio de la pregunta seis se estableció que el $100 \%$ de los encuestados están de acuerdo con que la apropiada gestión, la parroquia Julcuy puede potencializar el turismo cultural, por lo que la misma deberá ser fortalecida desde el GAD parroquial.

Mediante la pregunta siete fue posible conocer que el $30 \%$ están de acuerdo con que las entidades públicas están involucradas en el desarrollo turístico de la parroquia Julcuy; el $60 \%$ expreso estar en desacuerdo pues consideran que las entidades privadas deben de presentar un mayor interés en dicho proceso y el $10 \%$ expreso indecisión referente a la pregunta.

En lo que refiere a los servicios que se ofrece a los turistas son apropiados por medio de la pregunta nueve el 15\% expreso estar de acuerdo, el 55\% respondió estar en desacuerdo y el $30 \%$ restante expreso indecisión.

Por medio de la pregunta diez fue posible determinar que el $10 \%$ están de acuerdo en que las personas 0 prestadores de servicios turísticos de la parroquia Julcuy están capacitadas para realizar actividades turísticas; el $65 \%$ menciono estar en desacuerdo, dando a entender que no se encuentran adecuadamente capacitados y el $25 \%$ menciono estar indeciso, determinando que no conocen esta realidad.

\section{Conclusiones}

A través del análisis de los datos recolectados fue posible determinar la existencia de falencias relacionadas con los procesos de promoción turística de la parroquia Julcuy del cantón Jipijapa, entre las 
que se puede mencionar la no preservación de los sitios y lugares turísticos emblemáticos de la localidad, la falta de capacitación a los prestadores de servicios de la parroquia en atención al cliente. Se debe de promocionar de forma oportuna los recursos culturales y turísticos de la localidad y las entidades públicas deberán de alinearse a los procesos de gestión cultural en la parroquia.

A manera de conclusión, en cuanto a la información teórica, los resultados indican que contar y ejecutar un Sistema de Gestión de Calidad basada en las ISO 9001:2015 es una estrategia esencial para la promoción cultural de la parroquia Julcuy del cantón Jipijapa de la provincia Manabí.

\section{Bibliografía}

Almache Martínez, Z. R. (2013). Implementación de un sistema de gestión de la calidad para una empresa textil. Quito: Universidad Central del Ecuador. Obtenido de http://200.12.169.19/bitstrea $\mathrm{m} / 25000 / 1174 / 1 / \mathrm{T}-U C E-$ 0017-31.pdf

Amaya León, S. H. (2011). Propuesta de implementación de un sistema de gestión documental informatizado en la universidad Francisco Gavidia de El Salvador. El Salvador: Universidad Internacional de Andalucía.

Barona López, N. E., Estévez Bonilla, A. E., Tabares Burbano, v. P., \& Paredes Cruz, R. E. (2018). Sistema de gestión de calidad y metodología para la mejora continua en las curtidurías artesanales de la provincia del Tungurahua. Uniandes EPISTEME. Revista digital de Ciencia, Tecnología e Innovación. Obtenido de file:///C:/Users/CASA/Downlo ads/1522-4795-1-

PB\%20(1).pdf

Bojacá Beltrán, P. E. (2016). Propuesta de Modelo de Gestión para promover la cultura de la innovación en la IED Divino Salvador del Municipio de Cucunubá. Estudio de caso. Colombia: Universidad Tecnológica de Bolívar. Obtenido de https://biblioteca.utb.edu.co/n otas/tesis/0069861.pdf

Bonilla Velástegui, V. M. (2016). Manual de procedimientos para la gestión documental de la Subsecretaria Nacional de Gestión Pública de la secretaria de la administración del Ecuador y manejo de la información. Ambato - Ecuador: 
Universidad

Regional

Autónoma de los Andes "UNIANDES".

Brunner \& Catalán. (1987). Industria y mercado culturales en Chile: descripción y cuantificaciones.

(1.

FLACSO, Ed.) La Universidad de California. Obtenido de https://chart.googleapis.com/ chart?chs $=400 \times 400 \&$ cht $=$ qr \& chl=https://books.google.com .ec/books?id=2Na0AAAAIAA $\mathrm{J} \&$ source $=$ qrcode

Cadena Fernández, A. M. (2014). La abundancia de recursos humanos en la gestión administrativa. Cuenca: Escuela Superior Politécnica del Litoral. Obtenido de https://contenido.bce.fin.ec/d ocumentos/PublicacionesNot as/Catalogo/Cuestiones/XXII-04FERNANDEZ.pdf

Carmen Ballesteros. (2018). (N. 2. Gabriela Gracia ... VOL 15, Ed.) REVISTA LASALLISTA DE INVESTIGACIÓN-Vol. 15 No 2-2018-C. Ballesteros-367 $-377$.

Castellucci, D. (2011). Sistemas de calidad en turismo: posibilidades y restricciones de su implementación en Mar del Plata. Argentina: Universidad Nacional de Mar de Plata. Obtenido de http://nulan.mdp.edu.ar/1330/ 1/castellucci_di.pdf
Castro Segura, M. G., \& Muñoz Torres, J. A. (2017). Diseño de un plan estratégico que ayude a mejorar la gestión y competitividad, de los artesanos del programa Hidalgo del Desarrollo de la Ciudad de Guayaquil. Guayaquil - Ecuador: Universidad de Guayaquil. Obtenido de http://repositorio.ug.edu.ec/bit stream/redug/20607/1/\%E2\% 80\%9CDISE\%C3\%910\%20 DE\%20UN\%20PLAN\%20ES TRAT\%C3\%89GICO\%20QU E\%20AYUDE\%20A\%20MEJ ORAR\%20LA\%20GESTI\%C 3\%93N\%20Y\%20COMPETIT IVIDAD\%2C\%20DE\%20LOS $\% 20 A R T E S A N O S \% 20 . p d f$

Díaz Criollo, D. P. (2019). Modelo de gestión para el turismo cultural sostenible en la ciudad de Manizales. Manizales, Colombia: Universidad Nacional de Colombia. Obtenido de http://bdigital.unal.edu.co/718 57/1/1053832486.2019.pdf

Guadalupa Guerrero, E. D. (2012). Implementación de un sistema de gestión de calidad para el proyecto crecer del Gobierno Provincial del Azuay, según la normativa ISO 9001:2008. Cuenca: Universidad Politécnica Salesiana. Obtenido de https://dspace.ups.edu.ec/bits tream/123456789/7881/1/UP S-CT004726.pdf 
Guerrero Coloma, C. A. (2015). Diseño de un Modelo de Gestión de Talento Humano para Talleres Artesanales Madereros. Ambato Ecuador: Pontificia Universidad Católica del Ecuador Sede Ambato. Obtenido de https://repositorio.pucesa.edu .ec/bitstream/123456789/135 1/1/75738.pdf

Hernández Rueda, S. I., \& Monsalve Castro, C. (2015). Gestión de la calidad del servicio en la hotelería como elemento clave en el desarrollo de destinos turísticos sostenibles: caso Bucaramanga. Bogotá: Rev. esc.adm.neg no.78. Obtenido de

http://www.scielo.org.co/sciel o.php?script=sci_arttext\&pid= S0120-81602015000100011

Mendoza Torres, M. C. (2017). Modelo de gestión para fomentar el turismo rural en la zona centro de Manabí. Calceta: Escuela Superior Politécnica Agropecuaria de Manabí Manuel Félix López. Obtenido de http://repositorio.espam.edu.e c/bitstream/42000/499/1/TT6 6.pdf

Merino Jaramillo, J. A. (2014). La gestión cultural del Ecuador. Caso: Casa de la Cultura Ecuatoriana "Benjamín Carrión" 2008 - 2012. Quito:
Instituto de altos Estudios Nacionales. Obtenido de https://repositorio.iaen.edu.ec /bitstream/24000/3773/1/TES IS\%20FINAL.pdf

Pailiacho Mena, C. A. (2013). Diseño de un modelo de gestión turística sostenible para el aprovechamiento del patrimonio natural y cultural de la microcuenca del Río Chimborazo. Riobamba Ecuador: Escuela Superior Politécnica de Chimborazo. Obtenido de http://dspace.espoch.edu.ec/ bitstream/123456789/2764/1/ 23T0359.pdf

Pazmiño Ortiz, M. J. (2018). La promoción cultural del Ecuador a través de la gestión cultural de sus embajadas en el exterior. Quito: Universidad Central del Ecuador. Obtenido de http://www.dspace.uce.edu.e c/bitstream/25000/17358/1/TUCE-0013-CSH-059.pdf

Romero Donoso, D. P. (2016). Modelo para la gestión turística local sostenible vinculado a la Escuela Superior Politécnica Agropecuaria de Manabí "Manuel Félix López". Calceta: Escuela Superior Politécnica Agropecuaria de Manabí Manuel Félix López. Obtenido de http://repositorio.espam.edu.e 
c/bitstream/42000/338/1/TT5

9.pdf

Sarminto Iglesias, M. V. (2012).

Propuesta de promoción del potencial turístico de la

parroquia San José de

Ancón. Guayaquil:

Universidad Católica de

Santiago de Guayaquil.

Obtenido de

http://repositorio.ucsg.edu.ec/

bitstream/3317/295/1/T-

UCSG-PRE-ESP-AETH-

54.pdf

Unesco. (2021). Culturas ancestrales del Ecuador: lo femenino y lo masculino. (2. Museo Chileno de Arte Precolombino, Ed.) 71. Obtenido de https://chart.googleapis.com/ chart?chs $=400 \times 400 \&$ cht $=$ qr $\&$ chl=https://books.google.com .ec/books?id=0zOUAQAAIAA $\mathrm{J} \&$ source $=$ qrcode 